\title{
Single-Qubit Gates For Ensemble Qubits Using Off-Resonant Raman Interaction
}

\author{
R.A. Akhmedzhanov $v^{1,2,3}$, L.A. Gushchin ${ }^{1,2,3}$, A.A. Kalachev ${ }^{3}$, A.G. Litvak ${ }^{1,2}$, D.A. Sobgayda ${ }^{1,2,3}$, \\ and I.V. Zelensky, ${ }^{1,2,3 a}$ \\ ${ }^{1}$ Institute of Applied Physics of RAS, 46 Ulyanova Str., Nizhny Novgorod 603950, Russia \\ ${ }^{2}$ N.I. Lobachevsky Nizhny Novgorod State University, 23 Gagarina Ave., Nizhny Novgorod 603950, Russia \\ ${ }^{3}$ Kazan E.K. Zavoisky Physical-Technical Institute, 10/7 Sibirsky tract, Kazan 420029, Russia
}

\begin{abstract}
We analyze the single-qubit gates based on off-resonant Raman interaction when they are applied to qubits implemented as ensembles of active centers. We propose a new scheme which can be used to significantly decrease the influence of inhomogeneous broadening.
\end{abstract}

Keywords: quantum gate, Raman interaction, ensemble qubits

Realization of single-qubit quantum operations is the key factor for the development of the quantum calculation schemes [1]. Systems with their energy levels close to each other or even degenerate are often used as qubits, for such systems usually have the longest coherence times. In this case, it is convenient to use optical transitions to a certain auxiliary level in order to control the qubit state. Both individual active centers and ensembles of such centers are used as qubits. Specifically, one of the promising approaches is based on using ensembles of rare earth metal ions doped into dielectric crystals. Using qubit ensembles allows one to improve the signal-to-noise ratio, increase the efficiency of interaction with optical radiation, and simplify the technichal implementation. However, the inhomogeneous broadening of the ensemble, especially the inhomogeneous broadening of the optical transition used to control the qubit, becomes a significant factor that affects the quantum gate fidelity. Up to now, several ways of performing single-qubit gates using an optical transition to an auxiliary excited level have been suggested. One of the approaches is based on off-resonant Raman interaction $[2,3]$. In this case, pulses of bichromatic radiation are used to couple a given superpositional state to the excited level. The two field components are detuned from the resonance with their respective optical transitions. While these detunings are assumed to be significant, they are equal to each other, so the two-photon resonance condition is still satisfied (see figure 1a). In this case, the bichromatic field only interacts with a certain superposition of the qubit states - the so-called bright state $\mid \mathrm{b}>$ (see figure $1 \mathrm{~b}$ ), while the orthogonal dark state $\mid d>$ is not affected. When certain conditions are fulfilled, this problem can be considered adiabatically. If the excited state is initially empty, the bichromatic pulse application results in a phase shift $\varphi$ of the bright state. In order to implement an arbitrary unitary single qubit gate, one can adjust the relative intensities and phases of the bichromatic field

\footnotetext{
${ }^{\text {a }}$ Corresponding author: zelensky@appl.sci-nnov.ru
} 
components and the overall pulse energy so that the bright state coincides with an eigenvector of the operation and the phase $\varphi$ coincides with the required rotation angle.

In the present work we will consider using off-resonant Raman interaction to implement singlequbit gates for qubit ensembles, taking the inhomogeneous broadening of the ensemble into account. In this case, the qubit is defined as a group of particles with almost identical properties, differing only in the frequencies of their optical transitions to the excited level which are used to control the ensemble state. During the pulse sequence, particles from the inhomogeneously broadened absorption line acquire different overall phase shifts depending on their detunings $\delta$ from the center of the absorption line, which induces additional errors $\varphi_{\text {err }}$ in the resulting operation. It can be shown that for small detunings the phase error is linear, and the decrease in the fidelity is quadratic with respect to $\delta / \Delta$. This means that for sufficiently large detunings, the approach based on using off-resonant Raman interaction can be applied successfully to implement arbitrary single-qubit operations with qubit ensembles. However, increasing $\Delta$ leads to longer gate execution times, which limits the possibility of increasing the gate fidelity by increasing the detuning from the single-photon resonance.

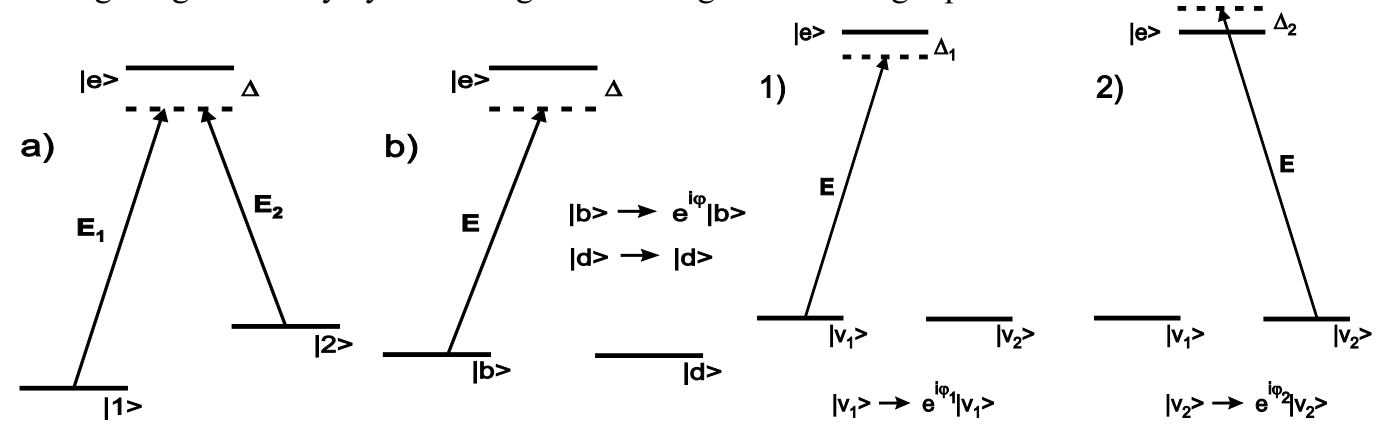

Figure 1. Left: single-qubit gate implementation scheme (a) in the energy basis $\mid 1>$ and $\mid 2>$ and (b) in the basis of the bright $\mid \mathrm{b}>$ and dark $\mid \mathrm{d}>$ states. As a result, the bright state acquires the phase shift $\varphi$.

Right: Single-qubit gate implementation scheme with two stages: (1) during the first stage, the eigenvector $\left|\mathrm{v}_{1}\right\rangle$ acquires the phase shift $\varphi_{1}$; (2) during the second stage, the eigenvector $\mid v_{2}>$ acquires the phase shift $\varphi_{2}$.

The main idea of the present work is to use two control pulses rather than one pulse as was proposed earlier (see figure 2). Each of these two pulses results in a phase shift for the respective eigenvectors of the desired single-qubit gate $-\varphi_{1}$ for the state $\left|v_{1}\right\rangle$ and $\left|v_{2}\right\rangle$ for $\varphi_{2}$. For the properly chosen parameters, this approach allows us to eliminate the linear term with respect to $\delta / \Delta$ in the phase error expression and, as a result, to improve the gate fidelity. In particular, this method can be implemented by choosing $\Delta_{1}=-\Delta_{2}, \varphi_{1}=-\varphi_{2}$.

Successful numerical simulations were performed to confirm our findings. System parameters were chosen according to the available data for qubits based on ensembles of rare earth ions doped into crystals. During these simulations it was also shown that the control pulses in our two-stage scheme can be applied both consecutively and simultaneously. Simultaneous pulse application can be used to reduce operation times.

The results were published in [4]. This work was supported by the Russian Science Foundation project 14-12-00806.

\section{References}

1. M.A. Nielsen, I.L. Chuang Quantum Computation and Quantum Information. (Cambridge: Cambridge University Press. 2001)

2. X. Caillet, C. Simon // Eur. Phys. J. D 42, P. 341-348 (2007)

3. E.M. Gauger, S.C. Benjamin, A. Nazir, B.W. Lovett, Phys. Rev. B. 77, P. 115322 (2008)

4. R.A. Akhmedzhanov, L.A. Gushchin, A.A. Kalachev, A.G Litvak, D.A. Sobgayda, I.V. Zelensky, Laser Phys. 25, P. 055202 (2015) 